\title{
DETECCIÓN DE RESIDUOS ANTIMICROBIANOS EN MÚSCULO, HÍGADO Y RIÑÓN DE CERDO EXPENDIDOS EN BOGOTÁ, COLOMBIA
}

\section{DETECTION OF ANTIMICROBIAL RESIDUES IN MUSCLE, LIVER AND KIDNEY OF PORK FOR SALE IN BOGOTA}

\author{
Sandra Ujueta Rodríguez ${ }^{1}$, Alejandra Araque Marín ${ }^{2}$
}

${ }^{1}$ MVZ, M.Sc. Microbiología, Docente investigador. Universidad de Ciencias Aplicadas y Ambientales, U.D.C.A, calle 222 No.5730, Bogotá, D.C., Colombia, e-mail: sujueta@udca.edu.co; ${ }^{2}$ MVZ, M.Sc. Farmacología, Docente investigador. U.D.C.A, e-mail: aaraque@udca.edu.co

Rev. U.D.C.A Act. \& Div. Cient. 19(2): 371-379, Julio-Diciembre, 2016

\section{RESUMEN}

El presente estudio, se orientó a identificar, preliminarmente, residuos de antimicrobianos en diferentes muestras de cerdo de expendios, de Bogotá, Colombia. Se realizó en dos etapas: en la primera, la identificación de residuos de betalactámicos, aminoglucósidos (estreptomicina) y sulfonamidas (sulfametazina), por el método microbiológico de cribado de las tres placas con Bacillus subtilis y, en la segunda, la tipificación de residuos de antimicrobianos de estos grupos, por un método comercial de ELISA, según los LMR, considerando muestras con residuos, aquellas que, por las dos pruebas, mostraron residuos de uno o varios de los antimicrobianos bajo estudio. De las 212 muestras estudiadas, el 33,0\% presentaron residuos de, por lo menos, un antimicrobiano. Para el hígado, de las muestras analizadas por las dos pruebas a betalactámicos, el $45,2 \%$ mostraron residuos; a estreptomicina, $10,8 \%$ y a sulfametazina, $28,6 \%$. De las muestras de riñón evaluadas resultaron con residuos de betalactámicos, el $44,8 \%$; de estreptomicina, $10,3 \%$ y sulfametazina, $23,1 \%$. De músculo, el 9,7\% presentaron residuos de betalactámicos; de estreptomicina, 0,0\% y para sulfametazina, la única muestra con este residuo por microbiología lo fue también por ELISA. Los resultados obtenidos por los métodos microbiológico y ELISA, que muestran una fuerza de concordancia considerable $(k>0,60)$, indican, de forma preliminar, un elevado porcentaje de muestras con residuos de antimicrobianos, lo cual, sustenta que el sacrificio de animales, sin respetar el tiempo de retiro, es frecuente y la necesidad de poner en marcha un plan de residuos, a lo largo de la cadena cárnica porcina.

Palabras clave: Residuos, antimicrobianos, producción porcina, fármacos.

\section{SUMMARY}

In this study, the presence of antimicrobials residues in pork tissues of markets, neighborhood groceries and butcher's shops in Bogota city (Colombia) was investigated. The study was performed following a two-step procedure: the first step consisting in the detection of beta-lactams, streptomycin and sulfamethazine traces using the microbiological method of the three plates with Bacillus subtilis; and the second step, following the commercial method ELISA for the samples showing a positive result. The samples were considered positive only if the presence of antimicrobials residues was confirmed by the two methods. The agreement in the results obtained by the two methods was also estimated $(\mathrm{k})$. Out of 212 samples analyzed, $33.0 \%$ were positive for at least one antimicrobial product. For liver samples, $45.2 \%$ were positive to beta-lactams, $10.8 \%$ to streptomycin and $28.6 \%$ to sulfamethazine. In the case of kidney $44.8 \%$ were positive to penicillin, $10.3 \%$ to streptomycin and $23.1 \%$ to sulfamethazine. Muscle showed a $9.7 \%$ positive result to beta-lactams, $0 \%$ to streptomycin and the only sample positive to sulfamethazine by the microbiological method, was also positive by the ELISA method. The results obtained by the two methods (microbiological and ELISA) were in agreement $(\mathrm{k}>0.60)$ and they indicate a high presence of antimicrobials in the commercialized pork tissues. This fact indicates that the slaughter of animals without respecting the time of retirement is a common practice and it shows the need to implement a waste plan throughout the pork meat chain.

Key words: Residually, antimicrobials, pig production, drugs.

\section{INTRODUCCIÓN}

En Colombia, la porcicultura ha evidenciado un proceso de expansión dentro de la economía nacional, con señales de 
dinamismo, reflejadas en el aumento sostenido del sacrificio (Díaz et al. 2011). La industria porcícola es uno de los sistemas de producción que emplea antimicrobianos y otros inhibidores, como terapéuticos profilácticos y como agentes promotores de crecimiento, los cuales, en dosis subterapéuticas, tienen la propiedad de mejorar la conversión del alimento, con aumentos diarios de peso, en el rango de 1 a $10 \%$ y producción de carnes de mejor calidad (Gaskins et al. 2002; Errecalde, 2004). Entre los efectos benéficos del uso de estos promotores de crecimiento, se encuentran la reducción de la morbilidad y la mortalidad en cerdos en crecimiento, mejoras en la tasa de partos, tamaño de la camada, peso al nacer y número de cerdos destetos por camada. También muestran eficacia en la reducción de la incidencia de mastitis, de metritis y de agalactia (Cromwell et al. 1996). De acuerdo con la OMS (2005), la mitad de todos los antimicrobianos producidos están destinados al consumo humano, mientras que el $50 \%$ restante, se usa para tratar a los animales enfermos o como promotores de crecimiento.

Es evidente que la resistencia antimicrobiana es promovida por el volumen de uso de los agentes antimicrobianos, cuyo amplio uso es reflejo de la elevada prescripción y del fácil acceso a estos medicamentos, a través de ventas en almacén y por la Internet, como formas de comercialización, que se han generalizado en muchos países, incrementando, en el mundo, el uso de antimicrobianos en humanos, en animales y en la agricultura, a pesar de las medidas adoptadas por algunos Estados. El incremento previsto de la demanda de productos alimenticios de origen animal puede llevar a aumentar el uso de antimicrobianos (OMS, 2015).

La cría de cerdos, se ha identificado como uno de los sectores en el que el uso de antimicrobianos conduce al desarrollo de resistencia (Rushton et al. 2014; Schwarz et al. 2001). La utilización amplia e indiscriminada de antimicrobianos en cerdos presenta varios inconvenientes, entre los cuales, el riesgo más importante para la salud de los consumidores, lo constituye el desarrollo de resistencia antimicrobiana, que implica, un riesgo de transferencia de microorganismos patógenos resistentes de los animales al hombre o de los genes que codifican resistencia de microorganismos patógenos o no patógenos a otros, en ocasiones patógenos, que pueden infectar al hombre, además de provocar, en los animales, fallas en tratamientos veterinarios (Franco et al. 1990; Jones, 2010).

La OIE reconoce que la resistencia a los agentes antimicrobianos constituye una preocupación mundial, ya que la utilización de estos productos en el hombre y en los animales, entre otros, tiene repercusiones sobre la salud pública y la sanidad animal (OIE, 2015).

Entre otros efectos adversos de los residuos de antimicrobianos, particularmente, los provocados por betalactámicos, sulfonamidas y aminoglucósidos, se encuentran el desarrollo de alergias e hipersensibilidad, de acuerdo con lo reportado por Cerniglia \& Kotarski (2005), así como el posible efecto carcinogénico y de hiperactividad de la sulfametazina, por lo cual, han sido considerados por el comité mixto FAO/ OMS de expertos, en aditivos alimentarios (JECFA, 1995), como factores determinantes para la evaluación y el establecimiento de niveles de residuos seguros en alimentos (JECFA, 1995; 1996).

Para la detección de residuos antimicrobianos, se suelen emplear métodos microbiológicos, inmunológicos o físicoquímicos. Los métodos microbiológicos de cribado, que han sido objeto de varias modificaciones (Nouws \& Ziv, 1979; Ebrecht, 1982; Nouws et al. 1988), desde la técnica descrita por Levetzow (1971) hasta los kits comerciales, actualmente disponibles en el mercado (Pikkemaat et al. 2008; 2011), son frecuentemente empleados para monitorear residuos de antimicrobianos en tejidos de animales sacrificados (Cornet et al. 2005), por que permiten cubrir un amplio espectro de antimicrobianos, analizar un elevado número de muestras y no requieren de equipamiento sofisticado.

En particular, la prueba de inhibición del crecimiento con $\mathrm{Ba}$ cillus subtilis BGA a diferentes niveles de $\mathrm{pH}$, para aumentar el espectro de captación de antimicrobianos y la adición de trimetoprim, para potenciar la acción de sulfonamidas, ha sido considerada una prueba útil, como alternativa para detectar una concentración mínima de antimicrobianos de los grupos betalactámicos, aminoglucósidos y sulfonamidas, en tejidos de bovinos y de porcinos sacrificados, que combina la sensibilidad a un amplio rango de antimicrobianos con el bajo costo, la practicidad y la obtención de resultados en corto tiempo (Pikkemaat et al. 2008; 2011). También son ampliamente aplicados los métodos inmunológicos semicuantitativos tipo ELISA, para el análisis inicial de residuos de antimicrobianos en tejidos, debido a que permiten analizar, simultáneamente, un gran número de muestras, por su bajo costo y facilidad operativa. Aunque la especificidad y los límites de detección dependerán del antibiótico a analizar, la extracción y limpieza que se haga a la muestra, estos métodos tienen, como ventaja importante, la objetividad con la automatización de la lectura (Reig \& Toldrá, 2008).

Colombia, se rige por los lineamientos del Codex Alimentarius, como instancia orientadora en materia de inocuidad de alimentos para la organización Mundial del Comercio (OMC), que establece los LMR en cerdo, para sulfonamidas en hígado, en riñón y en músculo, de 100ppb $(\mu \mathrm{g} / \mathrm{kg})$; para betalactámicos, de 50ppb $(\mu \mathrm{g} / \mathrm{kg})$ y para estreptomicina+dihid roestreptomicina, 600ppb $(\mu \mathrm{g} / \mathrm{kg})$, en músculo y en hígado y 1000ppb $(\mu \mathrm{g} / \mathrm{kg})$, en riñón (Resolución 1382 MinSalud y Protección Social, 2013). 
Puesto que en el país no existe el conocimiento sobre la presencia o niveles de residuos de antimicrobianos en el industria porcícola (Clavijo \& Donado, 2012) y uno de los retos importantes de la Cadena Cárnica Porcina es garantizar al consumidor final carne de cerdo inocua (Melo, 2013) y de calidad, el Consejo Nacional de Política Económica y Social de Colombia (Conpes 3458, 2007), que reconoce la carencia de una línea base de caracterización del estatus sanitario respecto a residuos, incluye, en la agenda prioritaria de investigación, establecer los niveles de residuos de medicamentos veterinarios en la carne de cerdo y en los productos cárnicos derivados, con el fin de mejorar las condiciones de sanidad e inocuidad de la cadena, aumentar la competitividad y fortalecer la capacidad de comercialización del país.

El presente estudio buscó aportar información sobre la presencia de residuos de betalactámicos, estreptomicina (aminoglucósidos) y sulfametazina (sulfonamidas) en músculo, en hígado y en riñón de cerdo, ofrecidos a la venta en Bogotá, mediante el empleo de dos pruebas de cribado, una microbiológica y otra ELISA.

\section{MATERIALES Y MÉTODOS}

El presente estudio, realizado entre enero de 2014 y febrero de 2015, fue de tipo descriptivo; se llevó a cabo en muestras de músculo, de hígado y de riñón de cerdo recolectadas, aplicando muestreo no probabilístico por conveniencia, en expendios de Bogotá, Colombia, teniendo en cuenta, al momento del muestreo, que se ofrecieran a la venta, como mínimo dos de estas tres partes, aptas para el consumo humano, conservadas en refrigeración y que provinieran de plantas de beneficio, autorizadas de la ciudad.

Las 72 porciones de músculo, los 73 hígados y los 67 riñones de cerdo, obtenidos en tres tipos de expendios, de frigorífico, de plaza de mercado y de barrio, ubicados en las nueve zonas de la ciudad, fueron analizados en el Laboratorio de Microbiología de la Universidad de Ciencias Aplicadas y Ambientales, U.D.C.A.

Para la detección de residuos de antimicrobianos, las muestras se analizaron, inicialmente, por la prueba microbiológica de inhibición, empleando Bacillus subtilis ATCC6633, para detectar la presencia de betalactámicos, aminoglucósidos y sulfonamidas (Gesche \& Mayor, 1990), posteriormente, se analizaron la totalidad de las muestras por la prueba comercial de ELISA Randox®, para betalactámicos, así como muestras que se identificaron con/sin presencia de residuos de aminoglucosidos y sulfonamidas, por las pruebas de ELISA (Randox $\left.{ }^{\circledR}\right)$, para estreptomicina-dihidroestreptomicina y para sulfametazina Se consideraron muestras con presencia de residuos aquellas que fueron identificadas con residuos de uno o varios de los antimicrobianos, estudiados por las dos pruebas.
El grado de concordancia entre los resultados de la prueba microbiológica y los de ELISA, definidos con o sin presencia del residuo por tipo de muestra, se determinó mediante el coeficiente kappa $(k)$, que se calculó según Landis \& Koch (1977), utilizando la siguiente fórmula:

$$
K=\frac{[(\Sigma \text { de concordancias observadas })-(\Sigma \text { de concordancias })]}{[(\text { No. Total de observaciones })-(\Sigma \text { de concordancias })]}
$$

Para la prueba de inhibición, las muestras de músculo, de hígado y de riñón, que se colectaron asépticamente, fueron identificadas y transportadas al laboratorio por separado, en bolsas de polietileno de cierre hermético, manteniéndolas en refrigeración a $5^{\circ} \mathrm{C}$. De cada muestra, se cortaron, con un sacabocados estéril, porciones cilíndricas de $8 \mathrm{~mm}$ de diámetro interno y $2 \mathrm{~mm}$ de espesor. Las porciones de músculo, se tomaron de cortes de un cuarto anterior o posterior; las de hígado, de un área distante a la vesícula biliar y las de riñón contenían, tanto médula como corteza renal (Levetzow et al. 1980).

La prueba microbiológica de inhibición siguió los lineamientos según Levetzow et al. (1980), con modificaciones propuestas por Nouws et al. (1988). Las porciones de las muestras fueron colocadas en cajas de petri con agar tripticasa soya, ajustado a pH 6,0; 7,2 y 8,0, luego de inoculado con esporas de $B$. subtilis, a una concentración de $10^{4}$ esporas/ $\mathrm{ml}$ de medio. Al medio ajustado a $\mathrm{pH} 7,2$ se le adicionaron $0,5 \mathrm{~mL}$ de trimetoprim, para la detección de sulfonamidas.

Para el control de la prueba, se utilizaron, como representantes de los grupos de antimicrobianos a detectar, sensidiscos de $6 \mathrm{~mm}$ de diámetro con penicilina G. 0,01 U.I en medio a pH 6,0; estreptomicina $0,5 \mathrm{mcg}$ en medio a pH 8,0 y sulfametazina $0,5 \mathrm{mcg}$ en medio a pH 7,2. Como controles negativos, se emplearon porciones de las muestras respectivas, provenientes de un lechón criado sin ningún tipo de tratamiento medicamentoso, que también fueron empleadas como controles negativos en las pruebas de ELISA.

Las porciones del músculo, del hígado y del riñón, se colocaron por duplicado sobre los medios de cultivo, a los tres niveles de $\mathrm{pH}$; luego, se incubaron por 18 horas a $30^{\circ} \mathrm{C}$; terminado el tiempo de incubación, se verificó que los discos control con antimicrobianos presentaran halos de inhibición de 5 a $10 \mathrm{~mm}$, para luego medir los halos de inhibición, observados en las muestras. La interpretación de resultados de la prueba de inhibición en placa, se consideró como positiva a la presencia del residuo, cuando se presentó un halo de inhibición del crecimiento, desde el borde del tejido hasta el inicio del crecimiento bacteriano de, al menos, $2 \mathrm{~mm}$; uno de 1 a $2 \mathrm{~mm}$, se consideró dudosa y uno menor a $1 \mathrm{~mm}$, negativa. Se calculó el promedio del ancho del halo de inhibición entre las correspondientes muestras duplicadas (Levetzow et 
al. 1980). Se descartaron las lecturas de las muestras que mostraron contaminación.

Para la prueba de ELISA, se emplearon los kits comerciales Randox® BL3448, STP 3468 y SM 2146, para betalactámicos, estreptomicina-dihidroestreptomicina y sulfametazina, respectivamente. Los homogenizados de las muestras para las pruebas de estreptomicina y de sulfametazina, se obtuvieron a partir de $1 \mathrm{~g}$ de tejido, al que se le adicionaron $9 \mathrm{~mL}$ de la solución de lavado respectivo. En el caso del homogenizado de la muestra para betaláctamicos, a $1 \mathrm{~g}$ de tejido, se adicionaron $9 \mathrm{~mL}$ de solución buffer fosfato $0,1 \mathrm{M} \mathrm{pH} 6,5$. La suspensión, se agitó en Vortex por 30seg y se centrifugó durante $10 \mathrm{~min}$, a $3000 \mathrm{rpm}$. Las suspensiones, se pasaron por filtros de nitrocelulosa para jeringa de $0,45 \mu \mathrm{l}$ y el sobrenadante obtenido, se diluyó en solución de lavado 1:1, para estreptomicina; $2: 1$, para betaláctamicos y para sulfametazina, no requirió dilución.

En las microplacas respectivas, se colocaron $50 \mu \mathrm{L}$ de la muestra a analizar por pozo y se adicionó el volumen de conjugado, indicado por el protocolo de cada prueba: $75 \mu \mathrm{L} /$ pozo, para estreptomicina y betalactámicos, $150 \mu \mathrm{L} /$ pozo, para sulfametazina; posteriormente, se incubaron las microplacas a $19^{\circ} \mathrm{C}$. La absorbancia de las muestras, se leyó a 450nm y la cantidad de antimicrobiano, se calculó sobre la base de la curva de calibración. El nivel de antimicrobiano, se expresó como $\mathrm{ng} / \mathrm{mL}$ del antimicrobiano en los tejidos analizados. El límite inferior de detección media para los antimicrobianos y las tasas de recuperación fueron mayores a $80 \%$ para todas las muestras. La prueba establece como límite de detección en tejido $5 \mathrm{ng} / \mathrm{g}$, para estreptomicina y sulfametazina y $10 \mathrm{ng} / \mathrm{g}$, para betalactámicos.

Los resultados obtenidos fueron comparados con los LMR establecidos por la Agencia Europea para la Evaluación de Medicamentos (Codex Alimentarius, 2012) y MinSalud y Protección Social de Colombia (2013), estableciéndose, como muestras con residuos, aquellas que presentaron residuos mayores o iguales para sulfametazina en hígado, en riñón y en músculo de 100ppb $(\mu \mathrm{g} / \mathrm{kg})$; para estreptomicina
$600 \mathrm{ppb}(\mu \mathrm{g} / \mathrm{kg})$, en músculo y en hígado y 1000ppb $(\mu \mathrm{g} / \mathrm{kg})$, en riñón y para betaláctamicos (penicilina $\mathrm{G}$, bencilpenicilina, bencilpenicilina procainica) 50ppb $(\mu \mathrm{g} / \mathrm{kg})$, en todos los órganos incluidos en el estudio.

\section{RESULTADOS Y DISCUSIÓN}

De las 212 muestras analizadas, inicialmente por la prueba microbiológica, cerca de la mitad $(43,4 \%)$ presentaron residuos de betalactámicos y en menor proporción residuos, tanto de aminoglucósidos como de sulfonamidas, siendo las vísceras las que revelaron mayor proporción de muestras con estos residuos, en comparación con el músculo y el riñón, la que presentó más muestras con residuos, comparada con el hígado (Tabla 1), resultado que coincide con la utilidad reconocida del riñón, para identificar, de forma rutinaria, residuos de antimicrobianos, debido a que éstos duran más tiempo en órganos que los excretan o metabolizan que en músculo y a su acumulación en este tejido, descrita por varios autores (Cantwell \& O'Keeffe, 2006; Orozco et al. 2006; FSIS, 2007).

Para residuos de betalactámicos, del total de muestras analizadas por las dos pruebas, cerca de una tercera parte $(33 \%)$ presentaron estos residuos, los cuales, superaron los límites permitidos, según resultados de la prueba de ELISA. Estos resultados coincidieron en identificar al hígado y al riñón como los tejidos que, además de presentar la mayor cantidad de muestras con presencia de residuos de betalactámicos, superan los límites permisibles de estos residuos, mientras que el músculo exhibió menor cantidad de muestras con residuos de este grupo (Tabla 2).

La presencia de residuos de betalactámicos en las muestras podría estar relacionada con su frecuente uso en la práctica médica veterinaria (Cartelle et al. 2014) e indicar la presencia de varios antimicrobianos del mismo grupo, como la penicilina G, amoxicilina y ampicilina, puesto que son incluidos en la identificación que hace la prueba de ELISA y corresponden con los antimicrobianos ampliamente utilizados, incluso, en combinaciones en el tratamiento de cerdos (Cameron-Veas et al. 2015).

Tabla 1. Muestras de cerdo con residuos de antimicrobianos identificados por prueba microbiológica con B. subtilis.

\begin{tabular}{|l|c|c|c|c|c|c|c|}
\hline & & \multicolumn{2}{|c|}{ Betalactámicos } & \multicolumn{2}{c|}{ Aminoglucósidos } & \multicolumn{2}{c|}{ Sulfonamidas } \\
\hline Tejido & $\begin{array}{c}\text { Muestras } \\
\text { analizadas }\end{array}$ & $\begin{array}{c}\text { \# de muestras } \\
\text { con residuos }\end{array}$ & $\%$ & $\begin{array}{c}\text { \# de mues- } \\
\text { tras con re- } \\
\text { siduos }\end{array}$ & $\%$ & $\begin{array}{c}\text { \# de muestras } \\
\text { con residuos }\end{array}$ & $\%$ \\
\hline Músculo & 72 & 8 & 11,1 & 0 & 0,0 & 1 & 1,4 \\
\hline Hígado & 73 & 40 & 54,8 & 5 & 6,8 & 6 & 8,2 \\
\hline Riñón & 67 & 44 & 65,7 & 8 & 11,9 & 6 & 9,0 \\
\hline
\end{tabular}


Tabla 2. Muestras de tejidos de cerdo con residuos de betalactámicos identificados por microbiología y ELISA.

\begin{tabular}{|c|c|c|c|c|c|c|c|}
\hline \multirow[b]{2}{*}{ Tejido } & \multirow[b]{2}{*}{$\begin{array}{c}\text { Muestras } \\
\text { analizadas }\end{array}$} & \multicolumn{2}{|c|}{ Microbiología } & \multicolumn{2}{|l|}{ ELISA } & \multicolumn{2}{|c|}{ Microbiología y ELISA } \\
\hline & & $\begin{array}{l}\text { Muestras } \\
\text { con resid- } \\
\text { uos }\end{array}$ & $\%$ & $\begin{array}{c}\text { Muestras con re- } \\
\text { siduos } \geq 50 \mathrm{ppb} \\
(\mu \mathrm{g} / \mathrm{kg})^{*}\end{array}$ & $\%$ & $\begin{array}{l}\text { Muestras con } \\
\text { residuos }\end{array}$ & $\%$ \\
\hline Músculo & 72 & 8 & 11,1 & 15 & 20,8 & 7 & 9,7 \\
\hline Hígado & 73 & 40 & 54,8 & 41 & 56,2 & 33 & 45,2 \\
\hline Riñón & 67 & 44 & 65,7 & 33 & 49,3 & 30 & 44,8 \\
\hline Totales & 212 & 92 & 43,4 & 89 & 42,0 & 70 & 33,0 \\
\hline
\end{tabular}

*LMR

Para estreptomicina, de las muestras analizadas por las dos pruebas (79), 10,1\% mostraron presencia de residuos que superan los límites máximos de este antibiótico, en los 3 tipos de tejidos estudiados. El tejido que mostró mayor número de muestras con residuos de aminoglucósidos por microbiología fue el riñón y, de esas muestras, la mitad indicaron residuos $\geq 1000 \mathrm{ppb}(\mu \mathrm{g} / \mathrm{kg})$, por ELISA. Para el hígado, la mayoría de las muestras que resultaron con residuos por la prueba microbiológica, resultaron concordantes por ELISA y las muestras de músculo que no presentaron residuos de estreptomicina fueron concordantes por las dos pruebas a este antibiótico (Tabla 3).

De los resultados para aminoglucósidos-estreptomicina que no fueron concordantes por las dos pruebas, se observó que la mayoría de resultados de muestras que presentaron residuos de este grupo de antimicrobianos fueron identificadas en riñón, por la prueba microbiológica, lo cual, se podría deber a la relativa baja sensibilidad a aminoglucósidos, que cita Vela et al. (2014), de esta prueba de inhibición. Según
Pikkemaat et al. (2011), la diferencia en la detección de aminoglucósidos estaría asociada, principalmente, con la matriz usada, detectando, en lugar de aminoglucósidos, constituyentes del tejido renal.

Para sulfonamidas, de las 48 muestras analizadas por microbiología y posteriormente por la prueba de ELISA para sulfametazina, cerca de una cuarta parte $(27,1 \%)$ de las muestras concordaron por las dos pruebas, siendo identificadas como muestras con presencia de estos residuos, por encima del límite máximo. El hígado y el riñón fueron los tejidos identificados con mayor proporción de muestras con residuos del antimicrobiano, mientras que la única muestra de músculo, identificada con residuos de sulfonamidas, por la prueba microbiológica, fue identificada también con residuos de sulfametazina por ELISA (Tabla 4). Los hallazgos de residuos en riñón coinciden con lo reportado por Medina et al. (2008), debido a que esta víscera concentra mayores cantidades de estos fármacos.

Tabla 3. Muestras de tejidos de cerdo con residuos de aminoglucósidos (estreptomicina) identificados por microbiología y ELISA.

\begin{tabular}{|l|c|c|c|c|c|c|c|}
\hline \multirow{2}{*}{ Tejido } & \multirow{2}{*}{$\begin{array}{c}\text { Muestras } \\
\text { analizadas }\end{array}$} & \multicolumn{2}{|c|}{ Microbiología } & \multicolumn{2}{c|}{ ELISA } & \multicolumn{2}{c|}{ Microbiología y ELISA } \\
\cline { 3 - 8 } & & $\begin{array}{c}\text { Muestras con } \\
\text { residuos }\end{array}$ & $\%$ & $\begin{array}{c}\text { Muestras con re- } \\
\text { siduos } \geq 600 \mathrm{ppb} \\
(\mu \mathrm{g} / \mathrm{kg})^{*}\end{array}$ & $\%$ & $\begin{array}{c}\text { Muestras con } \\
\text { residuos }\end{array}$ & $\%$ \\
\hline Músculo & 3 & 0 & 0,0 & 0 & 0,0 & 0 & 0,0 \\
\hline Hígado & 37 & 5 & 13,5 & 6 & 16,2 & 4 & 10,8 \\
\hline \multicolumn{1}{|c|}{ Riñón } & 39 & 8 & 20,5 & $\geq 1000 \mathrm{ppb}(\mu \mathrm{g} / \mathrm{kg})^{*}$ & 10,3 & 4 & 10,3 \\
\hline Totales & 79 & 13 & 16,5 & 10 & 12,7 & 8 & 10,1 \\
\hline
\end{tabular}


De las 13 muestras identificadas con residuos de sulfametazina, a excepción de una muestra de riñón en la que únicamente se detectó residuos de este antimicrobiano, las 12 muestras restantes ( 1 de músculo, 6 de hígado y 5 de riñón) presentaron, al mismo tiempo, residuos de sulfametazina y de betalactámicos, identificados por las dos pruebas. Ninguna de las muestras se identificó con residuos de sulfametazina y de estreptomicina, ni con los tres antimicrobianos por las dos pruebas.
En tres muestras de riñón y tres de hígado, se detectaron, por las dos pruebas, residuos de dos antimicrobianos, betalactámicos y estreptomicina, mientras que una muestra de hígado fue positiva, únicamente, a residuos de estreptomicina, por microbiología y ELISA, según LMRs (Codex Alimentarius).

En el estudio, el hígado y el riñón mostraron similar comportamiento, en cuanto a la proporción de muestras detectadas

Tabla 4. Muestras de tejidos de cerdo con residuos de sulfonamidas (sulfametazina) identificados por microbiología y ELISA.

\begin{tabular}{|l|c|c|c|c|c|c|c|}
\hline \multirow{2}{*}{ Tejido } & \multirow{2}{*}{$\begin{array}{c}\text { muestras } \\
\text { analizadas }\end{array}$} & \multicolumn{2}{|c|}{ Microbiología } & \multicolumn{2}{c|}{ ELISA } & \multicolumn{2}{c|}{ Microbiología y ELISA } \\
\cline { 3 - 8 } & $\begin{array}{c}\text { Muestras } \\
\text { con resid- } \\
\text { uos }\end{array}$ & $\%$ & $\begin{array}{c}\text { Muestras con re- } \\
\text { siduos } \geq 100 p p b \\
(\mu \mathbf{g} / \mathbf{k g})^{*}\end{array}$ & $\%$ & $\begin{array}{c}\text { Muestras con } \\
\text { residuos }\end{array}$ & $\%$ \\
\hline Músculo & 1 & 1 & 100,0 & 1 & 100,0 & 1 & 100,0 \\
\hline Hígado & 21 & 6 & 28,6 & 9 & 42,9 & 6 & 28,6 \\
\hline Riñón & 26 & 6 & 23,1 & 8 & 30,8 & 6 & 23,1 \\
\hline Totales & 48 & 13 & 27,1 & 18 & 37,5 & 13 & 27,1 \\
\hline
\end{tabular}

*LMR

con residuos de betalactámicos, estreptomicina y sulfametazina, por las dos pruebas.

El análisis de concordancia, coeficiente de concordancia CC y coeficiente de concordancia $k$ realizado entre los resultados de la prueba microbiológica y la prueba de ELISA, identificó una correspondencia considerable entre los resultados de las dos pruebas (Tabla 5). Para betalactámicos y estreptomicina, la prueba microbiológica y ELISA por separado identificaron más muestras positivas respecto a las muestras positivas corroboradas por las dos pruebas, lo cual, corresponde con un $k$ menor, mientras que para sulfametazina, el $k$ mayor correspondió con una mayor concordancia entre los resultados de las dos pruebas, para este antimicrobiano.

En general, el grupo de antimicrobiano que mostró la mayor proporción de muestras de tejidos de cerdo con residuos por encima de los LMR por las dos pruebas fue betalactámicos, seguido por sulfametazina y, por último, estreptomicina. Estos antimicrobianos identificados en las muestras, según la se encuentran entre los de mayor demanda, aprobados para uso en cerdos, siendo la penicilina, la amoxicilina y la ampicilina (betalactámicos), estreptomicina y dihidroestreptomicina (aminoglucósidos), y sulfametazina (sulfonamidas), empleados solos o en combinaciones de antibióticos estreptomicina+penicilina o combinaciones de sulfonami- das y antibióticos sulfametazina+penicilina+oxi o clortetraciclina, como promotores de crecimiento en cerdos en Colombia (ICA, 2014).

Los hallazgos de residuos de antimicrobianos, principalmente en las muestras de riñón y de hígado de cerdo analizadas, puede ser el resultado en la práctica veterinaria de no respetar el tiempo que debe transcurrir entre la última aplicación del medicamento y cuando el animal es llevado al sacrificio, definido como tiempo de retiro (Gustafson \& Bowen, 1997; Barton, 2000) que, dependiendo de la farmacocinética propia del medicamento, garantiza la inocuidad de la carne, de las vísceras y de los subproductos de origen animal, destinados al consumo humano. También, como resultado de regímenes terapéuticos que incluyan dosis repetitivas del medicamento (Medina et al. 2008), que se pueden acumular en reservorios tisulares o por sobredosis, aun estando por fuera del tiempo de retiro.

Las escasas muestras de músculo detectadas con antimicrobianos pueden evidenciar más un nivel farmacológico que un nivel de residuos (Koenen-Dierick, 1995; Medina et al. 2008), particularmente, bajo las condiciones actuales de los sistemas de explotación porcícola en las que, es de suponer, el uso de fármacos atiende los parámetros farmacocinéticos establecidos para la especie y los medicamentos son rápidamente eliminados del músculo. 
Tabla 5. Concordancia entre los resultados de la prueba microbiológica y la prueba de ELISA para residuos de antimicrobianos en muestras de cerdo.

\begin{tabular}{|l|c|c|c|}
\hline \multicolumn{1}{|c|}{ Antimicrobiano } & CC & k & Fuerza de concordancia \\
\hline Betalactámicos & 0,81 & 0,61 & Considerable \\
\hline Aminoglícósidos (estreptomicina) & 0,91 & 0,65 & Considerable \\
\hline Sulfonamidas (sulfametazina) & 0,9 & 0,76 & Considerable \\
\hline
\end{tabular}

La presencia considerable de residuos en los tejidos también se podría atribuir al uso de quimioterápicos, como promotores de crecimiento (Errecalde, 2004). Estos resultados llaman la atención sobre la necesidad de monitorear la presencia de residuos de antimicrobianos, puesto que el consumo de vísceras, que representa una opción nutricional de bajo costo en el país, puede exponer al consumo considerable de residuos que superan los límites permitidos, con el riesgo que esto representa para la salud pública.

Debido a que un método de cribado para detectar residuos de antimicrobianos requiere ser de bajo costo y alto rendimiento, capaz de identificar potenciales muestras no conformes, a partir de una gran cantidad de muestras (CCE CdICE, 2002; Stead et al. 2004), la prueba de inhibición con B. subtilis mostró ser utilidad en la detección de presencia de residuos de betalactámicos, aminoglucósidos y sulfonamidas, en tejidos de cerdo, por su considerable concordancia, con una técnica inmunológica semi-cuantitativa y por presentar un costo seis veces menor por muestra analizada, frente a esta técnica. Aunque a diferencia de lo citado en la literatura, la prueba microbiológica resultó ser más laboriosa y requerir mayor tiempo que la prueba de ELISA.

El empleo de la prueba de ELISA para la detección preliminar de residuos de antimicrobianos mostró, como ventajas, la capacidad de analizar un gran número de muestras en corto tiempo y presentar menor posibilidad de detectar falsos negativos; sin embargo, se requiere del uso de varios kits para cubrir una amplia gama de residuos antimicrobianos, lo cual, limita su uso, debido al mayor costo comparado con la prueba microbiológica, aunque representa una considerable reducción de costos y de esfuerzos dedicados a la identificación y cuantificación de antimicrobianos, por métodos confirmatorios.

Es imperioso para el país regularizar el uso de antimicrobianos en la producción porcícola, así como disponer de pruebas validadas para su seguimiento, poniendo en marcha el plan de residuos, que integre todas las actividades de la cadena porcícola, para prevenir, controlar y vigilar la presencia de estos residuos, que afectan la inocuidad del producto y la salud pública.
Conflicto de intereses: El presente trabajo fue preparado con la participación de todos los autores, quienes declaramos que no existe conflicto de intereses que ponga en riesgo la validez de los resultados o la voluntad de publicación de los mismos.

\section{BIBLIOGRAFÍA}

1. BARTON, M.D. 2000. Antibiotic use in animal feed and its impact on human health. Nutr. Res. Rev. 13:279299.

2. CAMERON-VEAS, K.; SOLA-GINES, M.; MORENO, M.; FRAILE, L.; MIGURA-GARCIA, L. 2015. Impact of the use of $\beta$-lactam antimicrobials on the emergence of Escherichia coli isolates resistant to cephalosporins under standard pig-rearing conditions. Appl. Environ. Microb. 81(5):1782-1787.

3. CANTWELL, H.; O'KEEFFE, M. 2006. Evaluation of the Premi ${ }^{\circledR}$ Test and comparison with the One-Plate Test for the detection of antimicrobials in kidney. Food. Adit. Contam. 23(2):120-125.

4. CARTELLE, M.; VILLACÍS, J.; ALULEMA CHICO, P. 2014. De la granja a la mesa. Implicaciones del uso de antibióticos en la crianza de animales para la resistencia microbiana y la salud. Rev. Cubana Aliment. Nutrición. 24(1):129-139.

5. CCE CdICE. 2002. Decisión de la Comisión 2002/657/ CE del 12 de agosto de 2002 por la que se aplica la Directiva 96/23/CE del Consejo en cuanto al funcionamiento de los métodos analítico y la interpretación de los resultados. Diario Oficial de las comunidades europeas. 17(221):8-36.

6. CERNIGLIA, C.; KOTARSKI, S. 2005. Approaches in the safety evaluations of veterinary antimicrobial agents in food to determine the effects on the human intestinal microflora. J. Vet. Pharmacol. Therapy. 28:3-20. 
7. CLAVIJO, V.; DONADO, P. 2012. Residuos de antimicrobianos en alimentos de origen animal. Rev. Porcicult. Col. 1(171):27-31.

8. CODEX ALIMENTARIUS. Límites Máximos de Residuos para medicamentos veterinarios en los alimentos. Actualizado en la $35^{\text {a }}$ sesión de la Comisión del Codex Alimentarius Commission; Disponible desde internet en http://www.ftp.fao.org/codex/weblinks/ MRL2_s_2012.pdf (con acceso el 07/2012).

9. CONSEJO NACIONAL DE POLÍTICA ECONÓMICA Y SOCIAL -CONPES-. 2007. República de Colombia, Departamento Nacional de Planeación, Documento Conpes 3458: Política nacional de sanidad e inocuidad para la cadena porcícola. 2007; Disponible desde Internet en: URL:http://www.dnp.gov.co/archivos/ documentos/Subdireccion_Conpes/3376.pdf (con acceso 25/08/2013).

10. CORNET, V.; GOVAERT, Y.; KOENEN-DIERICK, K.; OKERMAN, L.; DEGGROODT, J. 2005. Interlaboratory study based on a one-plate screening method for the detection of antibiotic residues in bovine kidney tissue. Food Addit. Contam. 22(5):415-422.

11. CROMWELL, G.; COFFEY, R.; AARON, D.; LINDEMANN, M.; PIERCE, J.; MONEGUE, H.; RUPARD, V.; COWEN, D.; PARIDO, M.; CLAYTON, T. 1996. Differences in growth rate of waling barrows and gilts. J. Anim. Sci. (Abstra.)74(Suppl. 1):186.

12. DÍAZ, C.; RODRIGUEZ, M.; VERA, V.; RAMIREZ, G.; CASAS, G.; MOGOLLON, J.; 2011. Caracterización de los sistemas de producción porcina en las principales regiones porcícolas colombianas. Rev, Col. Cie. Pec. 24:131-144.

13. EBRECHT, A. 1982. Verbesserung des Hemmstofftestes durch Zusatz von Trimetoprim zum Nachweis von Sulfonamiden. Arch. Lebensmittelhyg. 33:109-115.

14. ERRECALDE, J. 2004. Uso de antimicrobianos en animales de consumo. Estudio de incidencia del desarrollo de resistencias en salud pública. FAO. Roma. ftp://ftp.fao.org/docrep/fao/007y5468s/y5468s00. pdf 67p.

15. FRANCO, D.; WEBB, J.; TAYLOR, C. 1990. Antibiotic and Sulfonamide residues in meat: implications for human health. J Food Prot. 53:179-185.

16. FSIS. 2007. Bioassay for the Detection, Identification and Quantitation of Antimicrobial Residues in Meat and Poultry Tissue MLG 34.02. USDA, Food Safety and Inspection Service, Public Health Science. 60p.

17. GASKINS, H.R.; COLLIER, C.T.; ANDERSON, D.B. 2002. Antibiotics as growth promotants: mode of action. Anim. Biotechnol. 13(1):29-42.

18. GESCHE, E.; MAYOR, M. 1990. Sensibilidad de la técnica del Bacillus subtilis BGA en la detección de antimicrobianos. Arch. Med. Vet. 121p.

19. GUSTAFSON, R.H.; BOWEN, R.E. 1997. Antibiotic use in animal Agricultura. J. Appl. Microbiol. 83:531-541.

20. INSTITUTO COLOMBIANO AGROPECUARIO -ICA-. 2014. Productos medicamentos veterinarios con registro ICA vigente a febrero 20 de 2014. Bogotá. D.C. Subgerencia de protección animal; Disponible desde internet en http://www.ica.gov.co/CMSPages/ GetFile. aspx?nodeguid =49ab2753-6861-43f2b58a-e0adc1e5e797\&lang=es-CO (con acceso el 20/01/2015).

21. JECFA Joint FAO/WHO. 1995. Expert Committee on Food Additives. Evaluation of certain veterinary drug residues in food, WHO Technical Report Series $N^{\circ}$ 855, Geneve.

22. JECFA Joint FAO/WHO. 1996. Expert Committee on Food Additives, Evaluation of certain veterinary drug residues in food, WHO Technical Report Series $N^{\circ}$ 864, Geneve.

23. JONES, P. 2010. Medicamentos veterinarios: una necesidad imperiosa. Boletín de la Organización Mundial de la Salud Animal. Reino Unido. 1:3-5.

24. KOENEN-DIERICK, K. 1995. A one-plate microbiological screening test for antibiotic residue testing in kidney and meat: an alternative to the EEC four- plate method. Food. Addit. Contam. 12:77-82.

25. LANDIS, J.; KOCH, G. 1977. The measurement of observer agreement for categorical data. Biometrics. 33:159-74.

26. LEVETZOW, R. 1971: Untersuchengen auf Hemmstoffe im Rahme der bacteriologischen Fleischuntersuchung (BU). Bundesgesundheitsbl. 14:211-213.

27. LEVETZOW, R.; WEISE, E.; OTTO, D. 1980: Versuche zur Erweiterung des Nachweisspektrums beim biologischen Hemmstofftest. Bundesgesundheitsbl. 23:9-13. 
28. MEDINA, M.; GONZÁLEZ, D.; RAMÍREZ, A. 2008. Detección de residuos de antimicrobianos en tejidos comerciales y tetraciclinas en hueso de cerdo. Rev. Salud Anim. 30(2):110-115.

29. MELO, O. 2013. Manual de instrucciones para el manejo rentable de la carne de cerdo. Disponible desde Internet en: http://www.foodsafety.com.co/pdf/memorias/2013/LB/Manejo_inocuo_de_la_carne_de_cerdo_Oscar_Melo.pdf (con acceso 30/09/2014).

30. MINISTERIO DE SALUD Y PROTECCIÓN SOCIAL. 2013. Resolución 00001382 de 2 de mayo de 2013. República de Colombia. 15p.

31. NOUWS, J.; ZIV, G. 1979. A critical evaluation of several microbiological test methods for residues of antimicrobial drugs in ruminants. Arch. Lebensmittelhyg. $30: 4-8$.

32. NOUWS, J.; BROEX, N.; DEN HARTOG, J.; DRIESSENS, F. 1988. The New Dutch Kidney Test. Arch. Lebensmittelhyg. 39:133-156.

33. OIE. 2015. Introducción a las recomendaciones para controlar la resistencia a los agentes antimicrobianos. Código sanitario para los animales terrestres. Capítulo 6.6. Artículo 6.6.1. 30/07/2015.

34. OMS. 2005. Conferencia regional FAO/OMS sobre inocuidad de los alimentos para la Américas y el Caribe. Documento de sala No. 47. San José, Costa Rica, 6-9 de diciembre de 2005.

35. OMS. 2015. $68^{\mathrm{a}}$ Asamblea mundial de la salud. Resistencia a los antimicrobianos, Proyecto de plan de acción mundial sobre la resistencia a los antimicrobianos. 21p.

36. OROZCO, A.; AVALOS, E.; GONZALEZ, D.; PACHECO, C.; RAMIREZ, A.; KUHNE, M. 2006. Residuos de penicilina y tetraciclinas en cerdo. Avances en la Investigación Científica en el CUCBA. XVII Semana de la Investigación Científica. p.792-797.
37. PIKKEMAAT, M.G.; OOSTRA-VAN DIJK, S.; SCHOUTEN, S.; RAPALLINI, M.; VAN EGMOND, H.J. 2008. A new microbial screening method for the detection of antimicrobial residues in slaughter animals: The Nouws antibiotic test (NAT-screening). Food Control. 19(8):781-789.

38. PIKKEMAAT, M.G.; RAPALLINI, M.L.; ZUIDEMA, T.; ELFERINK, J.A.; OOSTRA-VAN DIJK, S.; DRIESSENVAN LANKVELD, W.D. 2011. Screening methods for the detection of antibiotic residues in slaughter animals: comparison of the European Union Four-Plate Test, the Nouws Antibiotic Test and the Premi ${ }^{\circledR}$ Test (applied to muscle and kidney). Food. Addit. Contam. 28(1):26-34.

39. REIG, R.; TOLDRÁ, F. 2008. Veterinary drug residues in meat: Concerns and rapid methods for detection. Meat Science. 78(1-2):60-67.

40. RUSHTON, J.; PINTO FERREIRA, J.; STÄRK, K.D. 2014. Antimicrobial Resistance: The Use of Antimicrobials in the Livestock Sector. OECD Publishing. Food, Agriculture and Fisheries Papers, No. 68. 44p.

41. SCHWARZ, S.; KEHRENBERG, C.; WALSH, T.R. 2001. Use of antimicrobial agents in veterinary medicine and food animal production. Int. J. Antimicrob. Agents. 17:431-437.

42. STEAD, S.; SHARMAN, M.; TARBIN, J.; GIBSON, E.; RICHMOND, S.; STARK, J. 2004. Meeting maximum residue limits: an improved screening technique for the rapid detection of antimicrobial residues in animal food products. Food. Add. Contam. 21(3):216219.

43. VELA, K.; TAFUR, M.C.; VELA, M.; SUÁREZ, M. 2014. Evaluación preliminar del bioensayo para la detección de antimicrobianos en músculo bovino. Rev. Fac. Química Farmacéut. 21(3):178-190.

Recibido: Septiembre 21 de 2015

Aceptado: Octubre 3 de 2016

Cómo citar:

Ujueta Rodríguez, S.; Araque Marín, A. 2016. Detección de residuos antimicrobianos en musculo, higado y riñon de cerdo expendidos en Bogotá, Colombia. Rev. U.D.C.A Act. \& Div. Cient. 19(2): 371-379. 